\title{
Zeta Converter with Resistor-capacitor-diode Snubber and H-type Snubber to Reduce Switching and Reverse-recovery Losses
}

\author{
Cheng-Tao Tsai* and Ji-Xin Chen \\ Department of Electrical Engineering, National Chin-Yi University of Technology, Taichung 41170, Taiwan
}

(Received June 27, 2020; accepted January 6, 2021)

Keywords: resistor-capacitor-diode, zero-voltage transition

In this paper, a zeta converter with a resistor-capacitor-diode (RCD) snubber and an H-type snubber to reduce switching and reverse-recovery losses is proposed. The proposed converter has the following advantages: (1) By incorporating an RCD snubber, the power switch can achieve a zero-voltage transition (ZVT) to reduce the switching losses of the power switch. (2) By incorporating an H-type snubber, the reverse-recovery losses of the power diode can be reduced. Therefore, the overall efficiency of the proposed converter is increased significantly. Finally, a prototype zeta converter with an RCD and an H-type snubber to reduce the switching losses of the power switch and the reverse-recovery losses of the power diode is built. Experimental results verify that the proposed zeta converter is relatively suitable for the requirements of a low or medium power.

\section{Introduction}

Dc-dc converters are switching power converters that convert a dc voltage to a different dc voltage, often providing a regulated output. Non-isolated switching power converters are classified by the relationship between the step-up and step-down voltages, for example, buck, boost, buck-boost, Cuk, SEPIC, and zeta converters. In the dc-dc applications of step-up or step-down voltages, the zeta converter is widely adopted, because the output voltage of the zeta converter can be higher or lower than the input voltage. To reduce the size and weight of components, the zeta converter is often operated at a high switching frequency. However, when operated at a high switching frequency, the power losses and electromagnetic interference (EMI) noise of the zeta converter are significantly increased. These disadvantages decrease conversion efficiency and power capability, which in turn seriously deteriorate the performance of the zeta converter. $^{(1-6)}$

To overcome the above disadvantages, zeta converters with soft-switching techniques to reduce power losses and EMI noise have been proposed. ${ }^{(7-12)}$ The soft-switching techniques can be divided into four techniques: zero-voltage switching (ZVS), zero-current switching (ZCS), zero-voltage transition (ZVT), and zero-current transition (ZCT). Among the four techniques, the ZVT technique has the simplest structure and easiest implementation for applications of

*Corresponding author: e-mail: cttsai@ncut.edu.tw https://doi.org/10.18494/SAM.2021.3022 
low- or medium-power converters. ${ }^{(12-16)}$ Zeta converters with the ZVT technique can effectively decrease the power losses of the power switch and EMI noise. A schematic diagram of the ZVT technique is illustrated in Fig. 1. In this study, a zeta converter is equipped with a resistorcapacitor-diode (RCD) snubber to reduce the switching losses of the power switch and with an H-type snubber to reduce the reverse-recovery losses of the power diode, as shown in Fig. $2 .^{(16-19)}$ The H-type snubber has a simple structure, consisting of a resonant inductor $\left(L_{1}\right)$, two resonant capacitors $\left(C_{1}\right.$ and $\left.C_{2}\right)$, and three diodes $\left(D_{1}, D_{2}\right.$, and $\left.D_{3}\right)$. The derivation of the H-type snubber is described in Sect. 2. The operational principles of the zeta converter with an RCD snubber and an H-type snubber are described in Sect. 3. Experimental results obtained from a prototype zeta converter are presented in Sect. 4. Finally, a conclusion is given in Sect. 5.

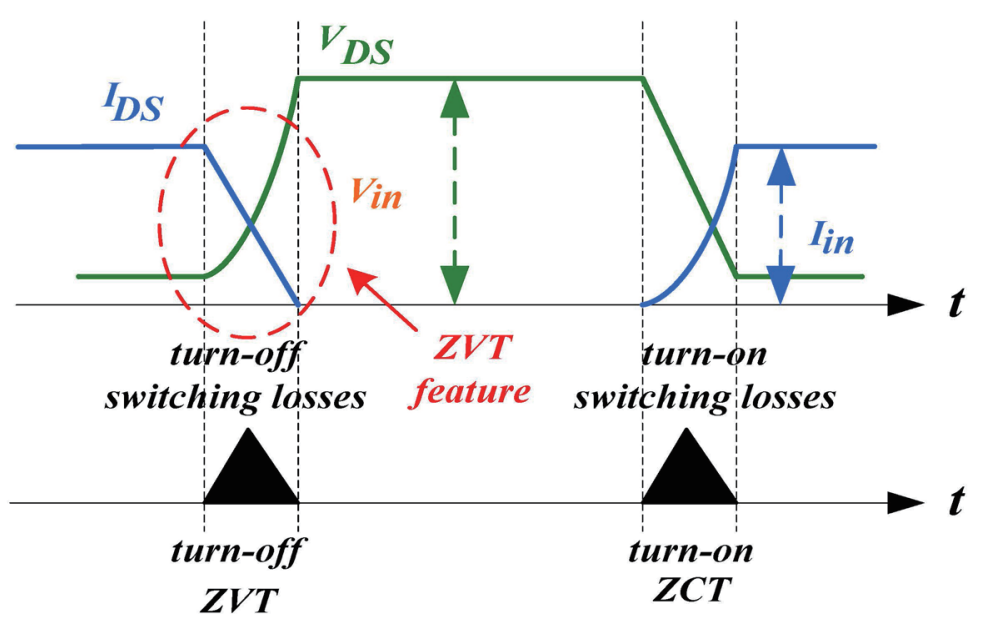

Fig. 1. (Color online) Illustration of ZVT technique.

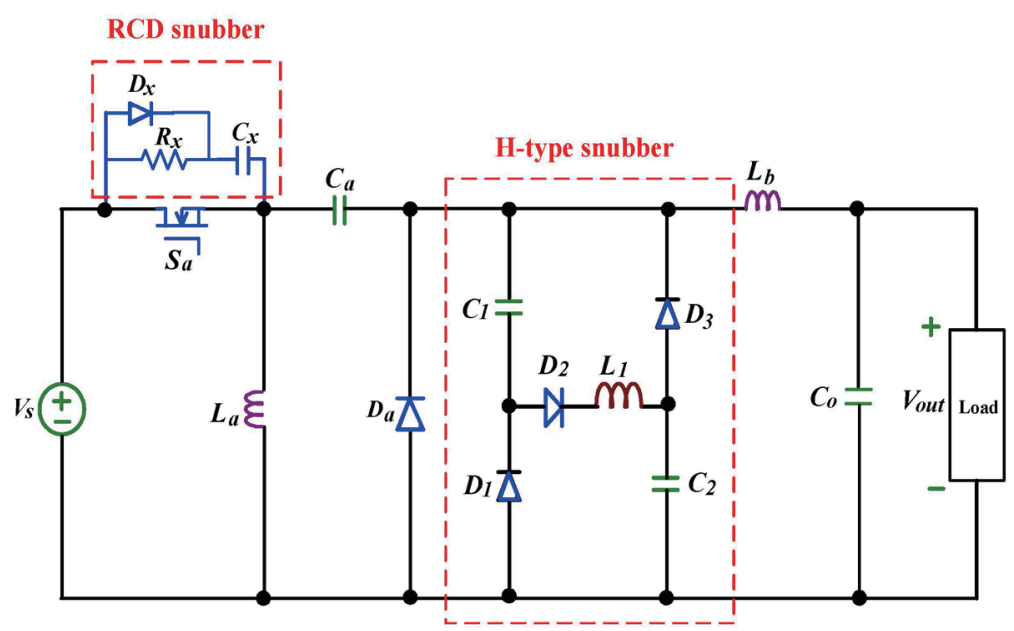

Fig. 2. (Color online) Topology of zeta converter with RCD snubber and H-type snubber to reduce switching and reverse-recovery losses. 


\section{Derivation of H-type Snubber}

The ZVT technique is applied by inserting a snubber cell in parallel with passive components (diodes, inductors, and capacitors) to limit $d v / d t$ of the drain-source voltage of the power switches. To illustrate the synthesis procedure, a zeta converter combining two possible snubber topologies is shown in Fig. 3. The two possible topologies have the same function of achieving the ZVT of the zeta converter. In Fig. 4(a), the two snubber topologies can utilize

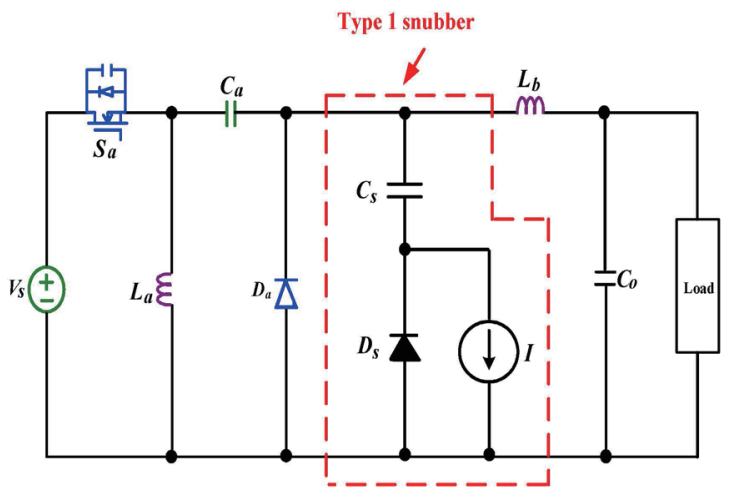

(a)

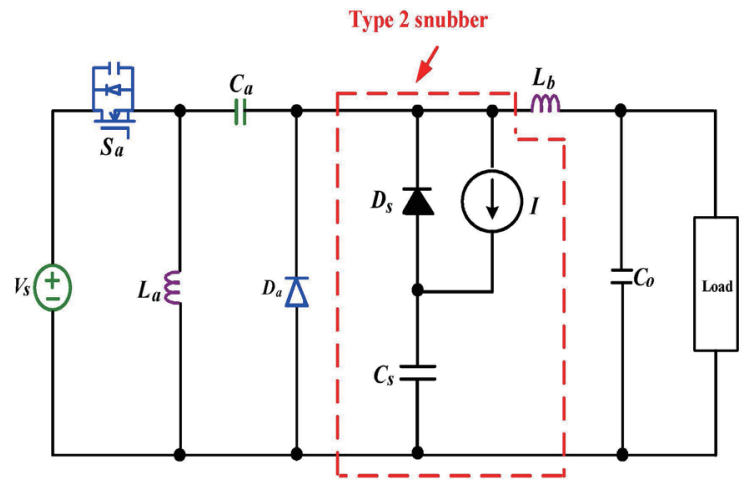

(b)

Fig. 3. (Color online) Equivalent topologies of zeta converter with (a) type 1 and (b) type 2 snubbers.
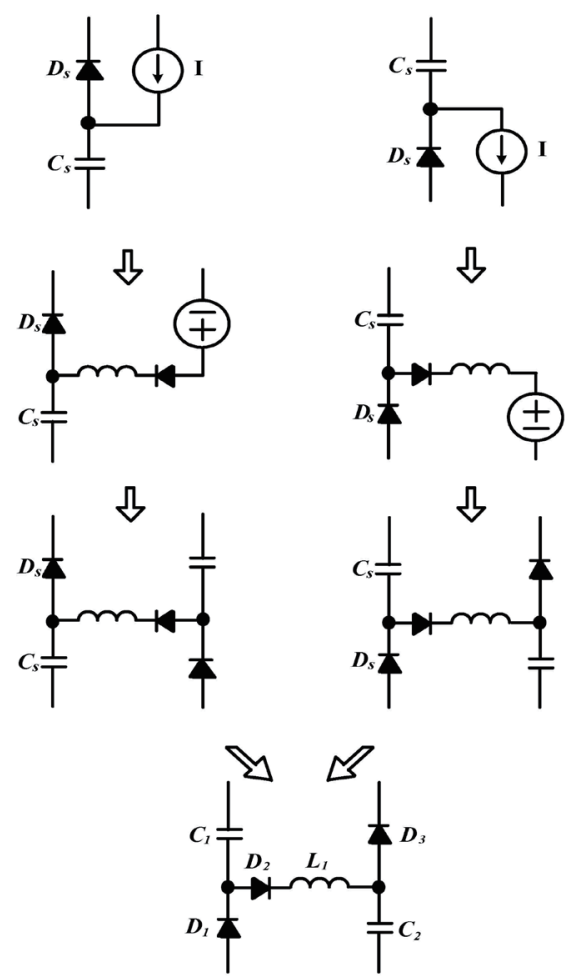

Fig. 4. Derivation of H-type snubber. 
the source transformation concept. Thus, the current source of each snubber topology can be identified as an inductor in series with a voltage source, as shown in Fig. 4(b). Each voltage source in Fig. 4(b) can be modeled as a capacitor connected in series with a diode, as shown in Fig. 4(c). Finally, an H-type snubber is derived, as shown in Fig. 4(d).

\section{Operational Principles}

Figure 2 shows the zeta converter with an RCD snubber to reduce the switching losses of the power switch $\left(S_{a}\right)$ and with an H-type snubber to reduce the reverse-recovery losses of the power diode $\left(D_{a}\right)$. The operational principles of the zeta converter over one switching cycle can be divided into four major operating modes. Figure 5 shows the current and voltage waveforms of the key components and the driving signal of the power switch $\left(S_{a}\right)$. Figure 6 shows the equivalent circuit modes of the zeta converter with an RCD snubber and an H-type snubber.

Mode 1 [Fig. 6(a), $t_{0}<t<t_{1}$ ]:

At time $t_{0}$, power switch $S_{a}$ is turned on and power diode $D_{a}$ is turned off. The inductor current $i_{L a}$ flowing through the path $V_{s} \rightarrow S_{a} \rightarrow L_{a} \rightarrow V_{s}$ is linearly increased. Simultaneously, the current of capacitor $C_{a}$ is divided into two paths, with some of the current flowing through the path $C_{a} \rightarrow C_{1} \rightarrow D_{1} \rightarrow L_{1} \rightarrow C_{2} \rightarrow V_{s} \rightarrow C_{a}$ and some of the current flowing through the path $C_{a} \rightarrow L_{b} \rightarrow$ Load $\rightarrow C_{a}$. During this interval, resonant inductor $L_{1}$ and resonant capacitors $C_{1}$ and

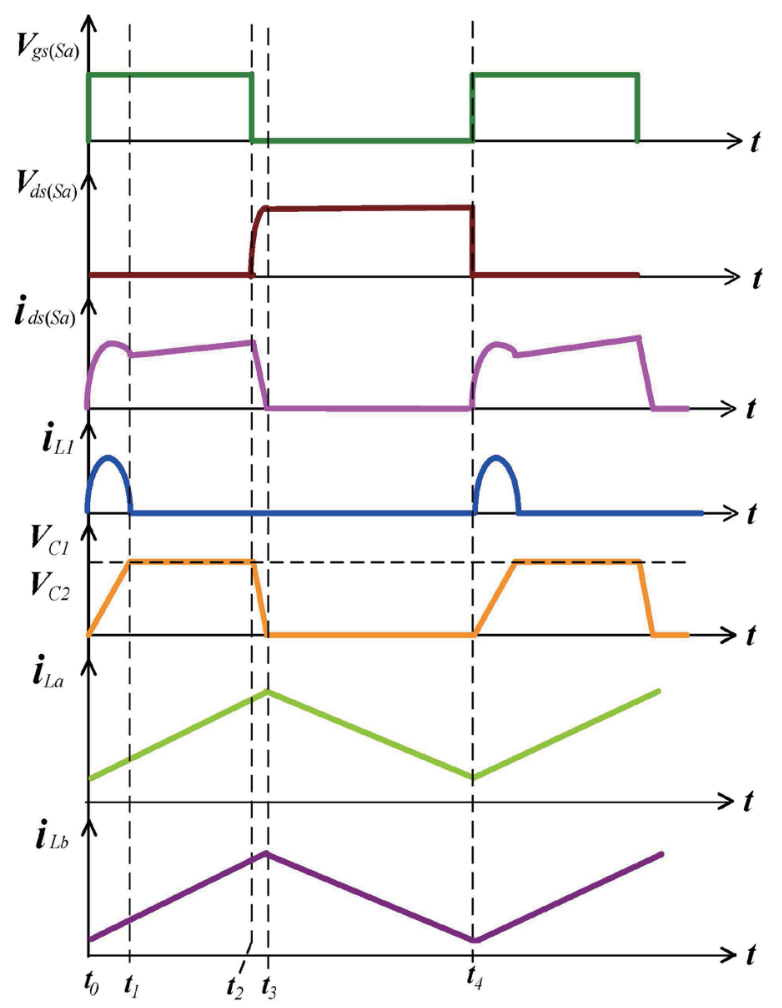

Fig. 5. (Color online) Current and voltage waveforms of key components for zeta converter with RCD snubber and H-type snubber. 


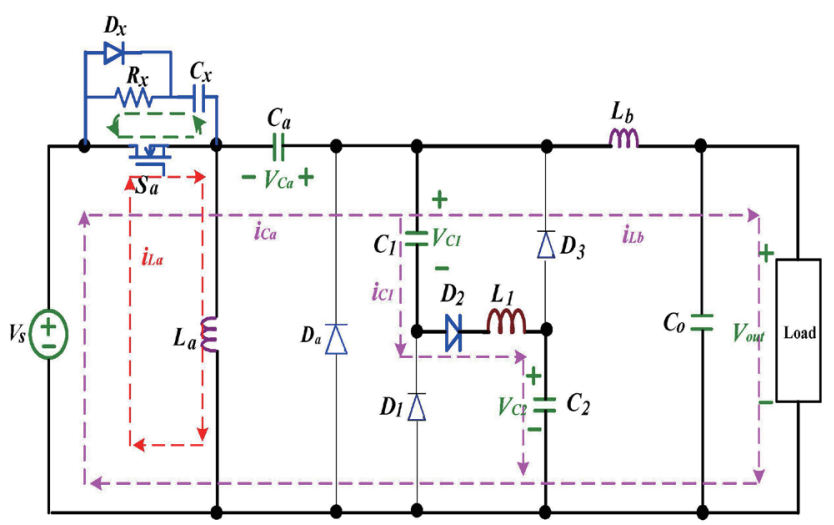

(a)

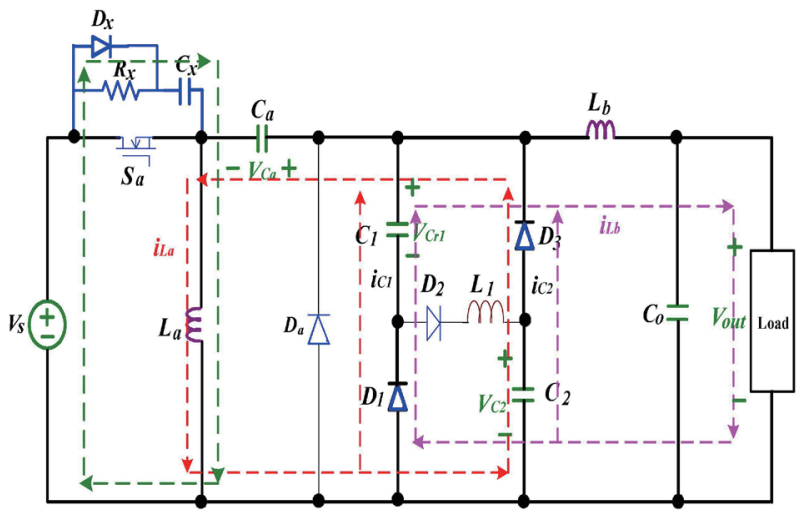

(c)

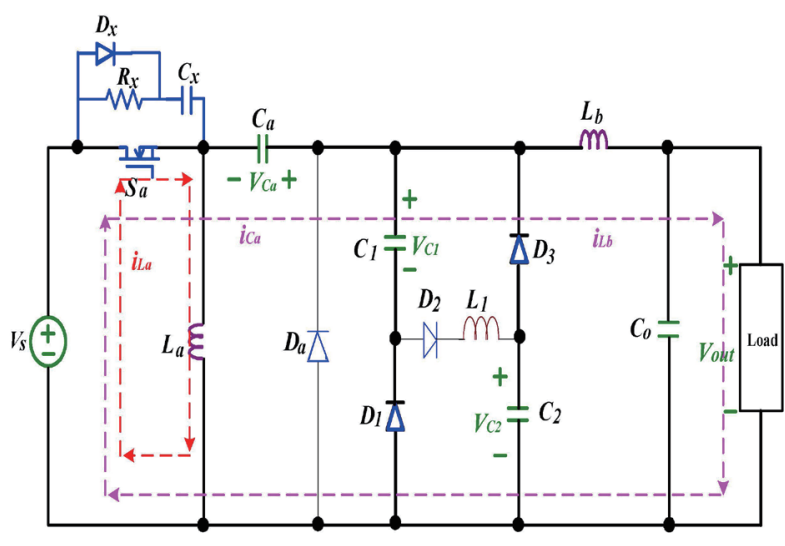

(b)

Fig. 6. (Color online) Equivalent circuits of zeta converter with RCD snubber and H-type snubber. (a) Mode 1. (b) Mode 2. (c) Mode 3. (d) Mode 4.

$C_{2}$ are charged in a resonant manner. The input current $i_{S}$ and the resonant current $i_{C a}$ can be expressed as

$$
i_{S}(t)=i_{L a}\left(t-t_{0}\right)+i_{C a}\left(t-t_{0}\right)
$$

and

$$
i_{C a}(t)=\left(V_{s}+V_{C a}\right) \sqrt{\frac{C}{2 L_{1}}} \sin \left[\omega_{0}\left(t-t_{0}\right)\right],
$$

where $C=2\left[\left(C_{1} C_{2} /\left(C_{1}+C_{2}\right)\right]\right.$ and $\omega_{0}=\sqrt{2 / L_{1} C}$. The equivalent circuit is shown in Fig. 6(a).

Mode 2 [Fig. 6(b), $t_{1}<t<t_{2}$ ]:

At time $t_{1}$, the voltages of the resonant capacitors $\left(V_{C 1}\right.$ and $\left.V_{C 2}\right)$ are equal to $V_{s}+V_{c a}$ and diodes $D_{1}$ and $D_{3}$ conduct. Simultaneously, the resonant current $i_{C 1}$ drops to zero and the resonant motion is completed. The currents $i_{L a}$ and $i_{L b}$ of the inductors can be expressed as 


$$
i_{L a}(t)=\frac{V_{s}}{L_{a}}\left(t-t_{1}\right)
$$

and

$$
i_{L b}(t)=\frac{V_{s}}{L_{a}}\left(t-t_{1}\right)
$$

The equivalent circuit is shown in Fig. 6(b).

\section{Mode 3 [Fig. 6(c), $t_{2}<t<t_{3}$ ]:}

At time $t_{2}$, power switch $S_{a}$ is turned off. The charges stored in capacitors $\mathrm{C}_{1}$ and $\mathrm{C}_{2}$ are discharged to the output through diodes $D_{1}$ and $D_{3}$, respectively. Thus, power switch $S_{a}$ is turned off under a ZVT condition. The currents $i_{L a}$ and $i_{L b}$ of inductors $L_{a}$ and $L_{b}$ can be expressed as

$$
i_{L a}(t)=i_{L b}(t)=\left(\frac{i_{C 1}+i_{C 2}}{2}\right)\left(t-t_{2}\right)
$$

The equivalent circuit is shown in Fig. 6(c).

Mode 4 [Fig. 6(d), $t_{3}<t<t_{4}$ ]:

At time $t_{3}$, the voltages of resonant capacitors $C_{1}$ and $C_{2}$ are discharged to zero and power diode $D_{a}$ conducts current. During this interval, the currents $i_{L a}$ and $i_{L b}$ of inductors $L_{a}$ and $L_{b}$ flow through power diode $D_{a}$, respectively. The currents $i_{L a}$ and $i_{L b}$ of inductors $L_{a}$ and $L_{b}$ can be expressed as

$$
i_{L a}(t)=\left(\frac{V_{C a}}{L_{a}}\right)\left(t-t_{3}\right)
$$

and

$$
i_{L b}(t)=\left(\frac{V_{o u t}}{L_{b}}\right)\left(t-t_{3}\right)
$$

The equivalent circuit is shown in Fig. 6(d). The operational mode of the zeta converter with an RCD snubber and an H-type snubber over one switching cycle is thus completed.

\section{Experimental Results}

To verify the feasibility of the zeta converter with an RCD snubber and an H-type snubber to reduce switching and reverse-recovery losses, a $100 \mathrm{~W}$ prototype is built. The specifications of the zeta converter are listed as follows: 
- input voltage: $V_{S}=120 \mathrm{~V}$,

- output voltage: $V_{\text {out }}=50 \mathrm{~V}$,

- output current: $I_{\text {out }}=2 \mathrm{~A}$,

- switching frequency: $f=50 \mathrm{kHz}$.

Figure 7 shows the experimental voltage and current waveforms of power switch $S_{a}$. It can be seen that the power switch has a ZVT feature at the turn-off transition. Therefore, the switching losses of the power switch can be reduced significantly. Figure 8 shows the experimental current waveforms of power diode $D_{a}$. It can be seen that the H-type snubber can reduce the reverserecovery losses of the power diode. The experimental efficiency of the zeta converter with an RCD snubber and an H-type snubber is shown in Fig. 9. It can be seen that the zeta converter can obtain a high efficiency (about 90\%).

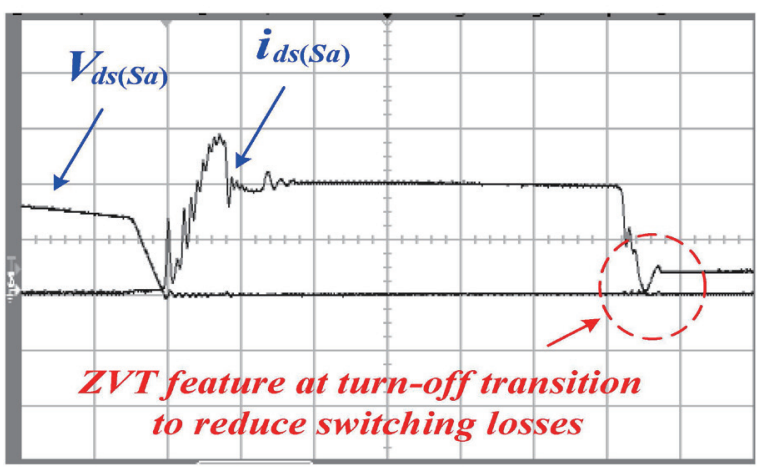

$\left(V_{d s(S a)}: 100 \mathrm{~V} / \mathrm{div}, i_{d s(S a)}: 2 \mathrm{~A} / \mathrm{div}\right.$, time: $\left.0.5 \mu \mathrm{s} / \mathrm{div}\right)$

Fig. 7. (Color online) Experimental voltage and current waveforms of power switch.

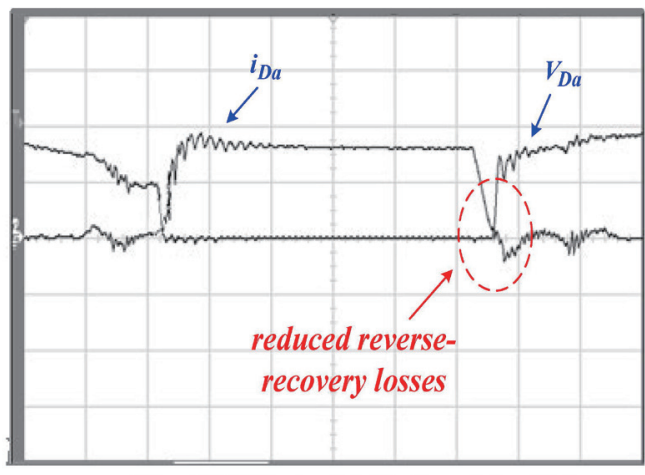

( $V_{D a}: 100 \mathrm{~V} / \mathrm{div}, i_{D a}: 2 \mathrm{~A} / \mathrm{div}$, time: $\left.0.5 \mu \mathrm{s} / \mathrm{div}\right)$

Fig. 8. (Color online) Experimental voltage and current waveforms of power diode.

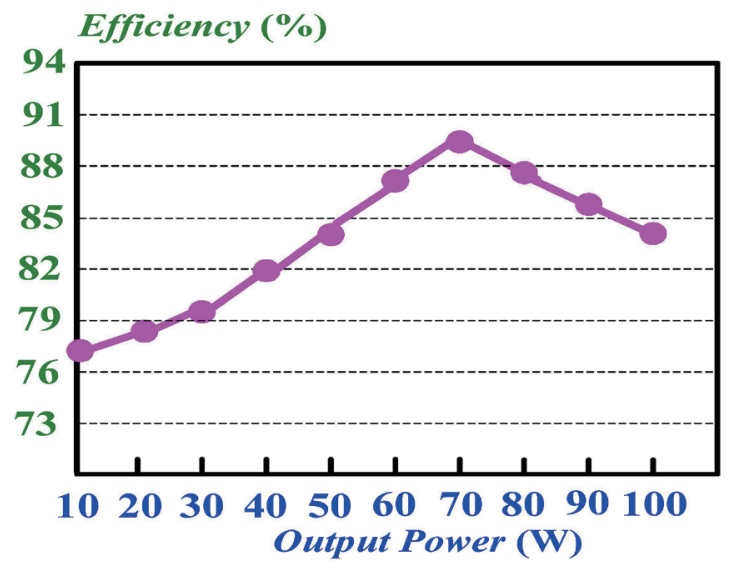

Fig. 9. (Color online) Efficiency of zeta converter with RCD snubber and H-type snubber to reduce switching and reverse-recovery losses. 


\section{Conclusion}

In this paper, a zeta converter with an RCD snubber and an H-type snubber to reduce switching and reverse-recovery losses has been built and implemented. Incorporating the simple RCD snubber and H-type snubber in the zeta converter can effectively reduce the power losses of the power switch $\left(S_{a}\right)$ and the reverse-recovery losses of the power diode $\left(D_{a}\right)$. Therefore, the overall efficiency of the zeta converter can be increased significantly. The experimental results verified that the zeta converter with an RCD snubber and an H-type snubber is relatively suitable for the requirements of a low or medium power.

\section{Acknowledgments}

This paper was supported by National Chin-Yi University of Technology, Taiwan.

\section{Author Contributions}

Both authors contributed to this paper. Cheng-Tao Tsai wrote the paper and Jin-Xin Chen contributed to the experimental results of the circuit.

\section{Conflicts of Interest}

The authors declare that there are no conflicts of interest regarding the publication of this paper.

\section{References}

1 Y. M. Chen, S. Y. Tseng, C. T. Tsai, and T. F. Wu: IEEE Trans. Aerospace Electron Syst. 40 (2004) 954. https:// doi.org/10.1109/TAES.2004.1337467

2 J. W. Kim, J. M. Choe, and J. S. J. Lai: IEEE Trans. Power Electron. 33 (2018) 4306. https://doi.org/10.1109/ TPEL.2017.2722985

3 T. J. Liang and J. H. Lee: IEEE Trans. Ind. Electron. 62 (2015) 4492. https://doi.org/10.1109/TIE.2014.2386284

4 H. Wang, H. S. Chung, and A. Ioinovici: IEEE Trans. Power Electron. 27 (2012) 2242. https://doi.org/10.1109/ TPEL.2011.2173588

5 A. Ajami, H. Ardi, and A. Farakhor: IEEE Trans. Power Electron. 30 (2015) 4255. https://doi.org/10.1109/ TPEL.2014.2360495

6 C. T. Tsai, Y. C. Kuo, Y. P. Kuo, and C. T. Hsieh: Energies 8 (2015) 1373. https://doi.org/10.3390/en8021373

7 P. Capaldi, A. Dannier, and I. Spina: IEEE Trans. Ind. Electron. 2 (2012) 374. https://doi.org/10.1109/ SPEEDAM.2012.6264623

8 H. Halbritter, C. Jager, R. Weber, M. Schwind, and F. Mollmer: IEEE Photo. Tech. Lett. 26 (2014) 1871. https:// doi.org/10.1109/LPT.2014.2336732

9 S. Y. Tseng: IEEE Trans. Ind. Electron. 55 (2008) 123. https://doi.org/10.1109/TIE.2007.896047

10 C. T. Tsai, J. C. Su, and S. R. Wei: Sens. Mater. 31 (2019) 327. https://doi.org/10.18494/SAM.2019.2086

11 S. W. Lee and H. L. Do: IEEE Trans. Power Electron. 32 (2017) 1375. https://doi.org/10.1109/ TPEL.2016.2549029

12 M. L. T. Nadia, S. Inoue, A. Kobayashi, and H. Akagi: IEEE Trans. Power Electron. 23 (2008) 2755. https:// doi.org/10.1109/TPEL.2008.2005388

13 J. Zhang, D. D.-C. Lu, and Ting Sun: IEEE Trans. Ind. Electron. 57 (2010) 1041. https://doi.org/10.1109/ TIE.2009.2028336

14 C. T. Tsai and C. L. Shen: IEEJ Trans. Elect. Electron. Eng. 8 (2013) 182. https://doi.org/10.1002/tee.21838 
15 C. T. Tsai, T. C. Liang, Y. C. Kuo, and Y. C. Luo: Comp. Electr. Eng. 5 (2016) 1373. https://doi.org/10.1016/ j.compeleceng.2016.01.010

16 T. F. Wu, Y. D. Chang, C. H. Chang, and J. G. Yang: IEEE Trans. Power Electron. 27 (2012) 1108. https://doi. org/10.1109/TPEL.2011.2126024

17 T. J. Liang and J. H. Lee: IEEE Trans. Ind. Electron. 62 (2015) 4492. https://doi.org/10.1109/TIE.2014.2386284

18 S. Jung and G. H. Cho: IEEE Trans. Ind. Electron. 61 (2014) 6710. https://doi.org/10.1109/TIE.2014.2316221

19 S. Y. Tseng and C. T. Tsai: Int. J. Photoenergy 2012 (2012) 1. https://doi.org/10.1155/2012/936843

\section{About the Authors}

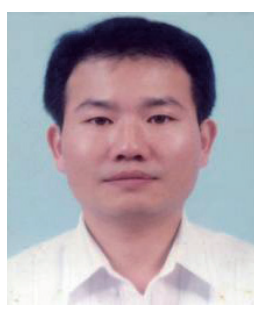

Cheng-Tao Tsai received his B.S. degree in electrical engineering from Feng Chia University, Taichung, Taiwan, in 1991 and his M.S. and Ph.D. degrees from National Chung Cheng University, Chia-Yi, Taiwan, in 2003 and 2008, respectively. Recently, he joined National Chin-Yi University of Technology, Taichung, Taiwan, where he is currently a professor in the Department of Electrical Engineering. His current research interests include the design of switching-mode power supplies, power factor correction technology, and chargers for electric vehicles.

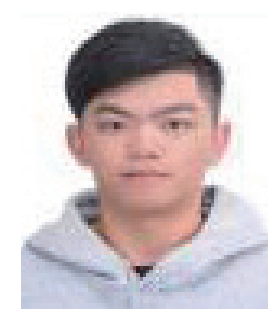

Ji-Xin Chen received his B.A. degree in electrical engineering in 2019 from National Chin-Yi University of Technology, Taichung, Taiwan. Currently, he is a graduate student. His area of research is switching-mode power supplies. 